Article

\title{
Enhancing and Tuning the Nonlinear Optical Response and Wavelength-Agile Strong Optical Limiting Action of $N$-octylamine Modified Fluorographenes
}

\author{
Aristeidis Stathis ${ }^{1,2}$, Michalis Stavrou ${ }^{1,2}$, Ioannis Papadakis ${ }^{1,2}{ }^{\infty}$, Ievgen Obratzov ${ }^{3}$ \\ and Stelios Couris $1,2, *$ (i) \\ 1 Department of Physics, University of Patras, 26504 Patras, Greece; a.stathis@iceht.forth.gr (A.S.); \\ m.stavrou@iceht.forth.gr (M.S.); j.papadakis@iceht.forth.gr (I.P.) \\ 2 Institute of Chemical Engineering Sciences (ICE-HT), Foundation for Research and \\ Technology-Hellas (FORTH), P.O. Box 1414, 26504 Patras, Greece \\ 3 Regional Centre of Advanced Technologies and Materials, Faculty of Science, Palacky University \\ in Olomouc, 77900 Olomouc, Czech Republic; ievgen.obraztsov@upol.cz \\ * Correspondence: couris@iceht.forth.gr
}

Received: 2 November 2020; Accepted: 20 November 2020; Published: 23 November 2020

\begin{abstract}
Fluorographene has been recently shown to be a suitable platform for synthesizing numerous graphene derivatives with desired properties. In that respect, $N$-octylamine-modified fluorographenes with variable degrees of functionalization are studied and their nonlinear optical properties are assessed using $4 \mathrm{~ns}$ pulses. A very strong enhancement of the nonlinear optical response and a very efficient optical limiting action are observed, being strongly dependent on the degree of functionalization of fluorographene. The observed enhanced response is attributed to the increasing number of defects because of the incorporation of N-heteroatoms in the graphitic network upon functionalization with $\mathrm{N}$-octylamine. The present work paves the way for the controlled covalent functionalization of graphene enabling a scalable access to a wide portfolio of graphene derivatives with custom-tailored properties.
\end{abstract}

Keywords: $\mathrm{N}$-doped graphene; nonlinear optical response; optical limiting; graphene functionalization

\section{Introduction}

In recent years, fluorographene [1,2] (FG) has become a template for the synthesis of new members of the graphene family [3-5], with controllable chemical and optical properties, suitable for a plethora of applications such as biosensing [6], gas sensing on surfaces [7] and solar cell technologies [8]. Unlike its parent, graphene, FG possesses great chemical activity because of the electronegative fluorine that attaches to carbon atoms along with the formation of various structural defects, placing FG among the suitable carbon precursors for the tunable and scalable synthesis of new graphene derivatives with desired properties $[9,10]$. Recent examples include thiol-, amine- and hydrogenated derivatives obtained by the partial nucleophilic substitution of FG by organic thiophenols (methoxy-thiophenol, dimethyl-amin-thiophenol), lithium diethylamide and H-, respectively [11-13]. The newly synthesized derivatives contain the selected ligand via substitution, resulting in the formation of $\mathrm{sp}^{2}$ nanodomains arising from the fluorine atoms' elimination, while they also contain some residual carbon-fluorine groups. Herein, we investigate the effect of the amine functionalization of FG via N-octylamine nucleophilic substitution on its nonlinear optical (NLO) response and optical limiting (OL) performance under nanosecond laser excitation. Doping FG with heteroatoms, such as nitrogen, can significantly 
alter its electronic structure due to tailored defect-induced states, leading to novel 2D materials whose optical and electronic properties can be controlled by modifying the amount of nitrogen $(\% \mathrm{~N})$ incorporated into the graphenic lattice. For instance, nitrogen doping can tune the electrical properties of some chemically functionalized graphenes, e.g., from a p-type semiconductor [14] to an n-type one [15]. In the present work, four FG derivatives, namely FG-OAx, with different $\mathrm{F}$ and $\mathrm{N}$ contents (ranging from $55 \%$ down to $2.3 \%$, and from $2.9 \%$ up to $8.1 \%$, respectively), were synthesized in order to demonstrate and quantify the effects of such functionalization on the NLO properties and $\mathrm{OL}$ action of the aforementioned derivatives. The atomic $\mathrm{F}$ and $\mathrm{N}$ content $(\mathrm{F} / \mathrm{N})$ was controlled by tweaking the reaction time of the $N$-octylamine (OA) ligand with FG. The investigated FG derivatives exhibited enhanced NLO responses compared to pristine FG and other covalently functionalized graphene derivatives, demonstrating the importance and effectiveness of graphene functionalization for superior NLO response and its tunability. As far as the OL action is concerned, the present derivatives were found to exhibit sizeable OL action in the range of 450 to $1950 \mathrm{~nm}$, operating more efficiently for visible wavelengths. The OL action of these FG-OAx derivatives arises mainly from their strong nonlinear absorption (NLA), which is significantly affected by the tailored defects of the derivatives. The present study demonstrates clearly that the heteroatom doping of FG is an efficient tool for the control of the NLO properties through controlled inducing defects. The further exploitation of these defect-engineered FGs can lead to materials suitable for various photonic and optoelectronic applications.

\section{Materials and Methods}

\subsection{Synthesis and Characterization}

Graphite fluoride, GrF (>61 wt. \% F, C1F1.1), was purchased from Sigma-Aldrich (St. Louis, MO, USA) and was used for the synthesis of pristine FG. The latter was obtained by the exfoliation of GrF in $N, N$-Dimethylformamide, or namely, DMF [16]. The four FG-OAx derivatives were prepared by partial nucleophilic substitution with the corresponding $N$-octylamine ligand, as previously described $[17,18]$. The alkylamine ligand acted as a nucleophile, attacking the electrophilic radical centers of FG, according to the recently explained pathway of the reactions of nucleophiles with FG [19] The reaction led to the covalent attachment of the octylamine molecules to the graphenic plane. The investigated samples were characterized by an arsenal of techniques, such as X-ray Photoelectron Spectroscopy (XPS), Fourier-transform infrared spectroscopy (FT-IR), Transmission Electron Microscopy (TEM) and Raman spectroscopy, to determine and confirm the successful functionalization of FG. The detailed synthesis along with the characterization results can be found in detail in references $[17,18]$. The XPS results have been previously reported and are presented here for the sake of better understanding in the context of explaining the photophysical properties. The four ( $N$-octylamine)-functionalized FG derivatives were named as FG-OA 20', FG-OA 30', FG-OA $6 \mathrm{~h}$ and FG-OA $24 \mathrm{~h}$, where the numbers indicate the reaction time. Raman spectra were acquired using a Dynamic Extended Resolution (DXR) Raman spectroscope (Thermo Scientific, Waltham, MA, USA) equipped with a laser operating at a wavelength of $633 \mathrm{~nm}$. The dried powder sample was deposited on the substrate, and the laser power on the sample was set to $2 \mathrm{~mW}$ and the exposure time was $2 \mathrm{~s}$. Each measured Raman spectrum represents an average of 1024 experimental microscans.

\subsection{Experimental Setups for the Measurements of the NLO Properties and the OL Performance}

The non-linear optical (NLO) response of the FG-octylamine (FG-OAx) derivatives was investigated by means of the Z-scan technique [20], employing visible $(532 \mathrm{~nm})$ and infrared $(1064 \mathrm{~nm}) 4 \mathrm{~ns}$ laser pulses. The Z-scan technique was selected because of its experimental simplicity and because it allows the simultaneous determination of both the magnitude and the sign of the nonlinear absorption, and the refraction of a sample from a single measurement. The nonlinear absorption and refraction of a sample are expressed by the nonlinear absorption coefficient $\beta$ and the nonlinear refractive parameter 
$\gamma^{\prime}$, which are related to the imaginary, $\operatorname{Im} \chi^{(3)}$, and real, $\operatorname{Re} \chi^{(3)}$, parts of the third-order nonlinear susceptibility, $\chi^{(3)}$. A detailed description of the experimental setup as well as the procedures followed for the analysis of the Z-scans data can be found in detail elsewhere [21]. For the Z-scan experiments, a 4 ns Q-switched Nd:YAG laser (EKSPLA NT 342/3/UVE/AW) was employed, operating at a repetition rate between 1 and $10 \mathrm{~Hz}$, at its fundamental (i.e., at $1064 \mathrm{~nm}$ ) or at its second harmonic frequency (i.e., at $532 \mathrm{~nm}$ ). For the optical limiting experiments, an Optical Parametric Oscillator (OPO) was used, pumped by the same Nd:YAG laser. In all cases, the laser beam was focused into the samples using a $20 \mathrm{~cm}$ focal length quartz plano-convex lens. Several dispersions with appropriate concentrations in order to exhibit the same linear transmittance, i.e., of $\sim 70 \%$, at each excitation wavelength used (i.e., at $450,532,650,750,950,1064,1250,1550$ and $1750 \mathrm{~nm}$ ) were prepared. The corresponding spot radii at the focus were determined using a charge-coupled device (CCD) camera, to achieve the incident laser intensity and/or the laser fluence needed for the experiments. The prepared dispersions of each FG-octylamine derivative were placed in $1 \mathrm{~mm}$ thick quartz cells for the optical measurements. For the Z-scan measurements performed at 532 and $1064 \mathrm{~nm}$, the corresponding Rayleigh length (i.e., confocal parameter) was determined. They were about 2 and $2.6 \mathrm{~mm}$, respectively, thus fulfilling the Z-scan condition concerning the thin sample approximation. For comparison purposes, some $\mathrm{C}_{60}$ toluene solutions were also prepared and used as reference during the evaluation of the OL performance, since $\mathrm{C}_{60}$ is generally considered as a benchmark material for OL studies.

\section{Results and Discussion}

\subsection{Measurements of the NLO Properties}

Some representative UV-Vis-NIR absorption spectra of FG and the four octylamine-functionalized FGs (FG-OAx), all dispersed in dichloromethane (DCM), are presented in Figure 1. As shown, FG exhibits the typical graphene featureless structure, whereas the spectra of the FG-OAxs exhibit an increasing absorption towards shorter wavelengths and a clearly distinct absorption feature between 250 and $265 \mathrm{~nm}$, ascribed to the $\mathrm{sp}^{2}$-conjugated carbons emerging from the reductive defluorination of FG $[22,23]$. The colored arrows indicate schematically the excitation wavelengths where the optical limiting (OL) action of the dispersions was evaluated.

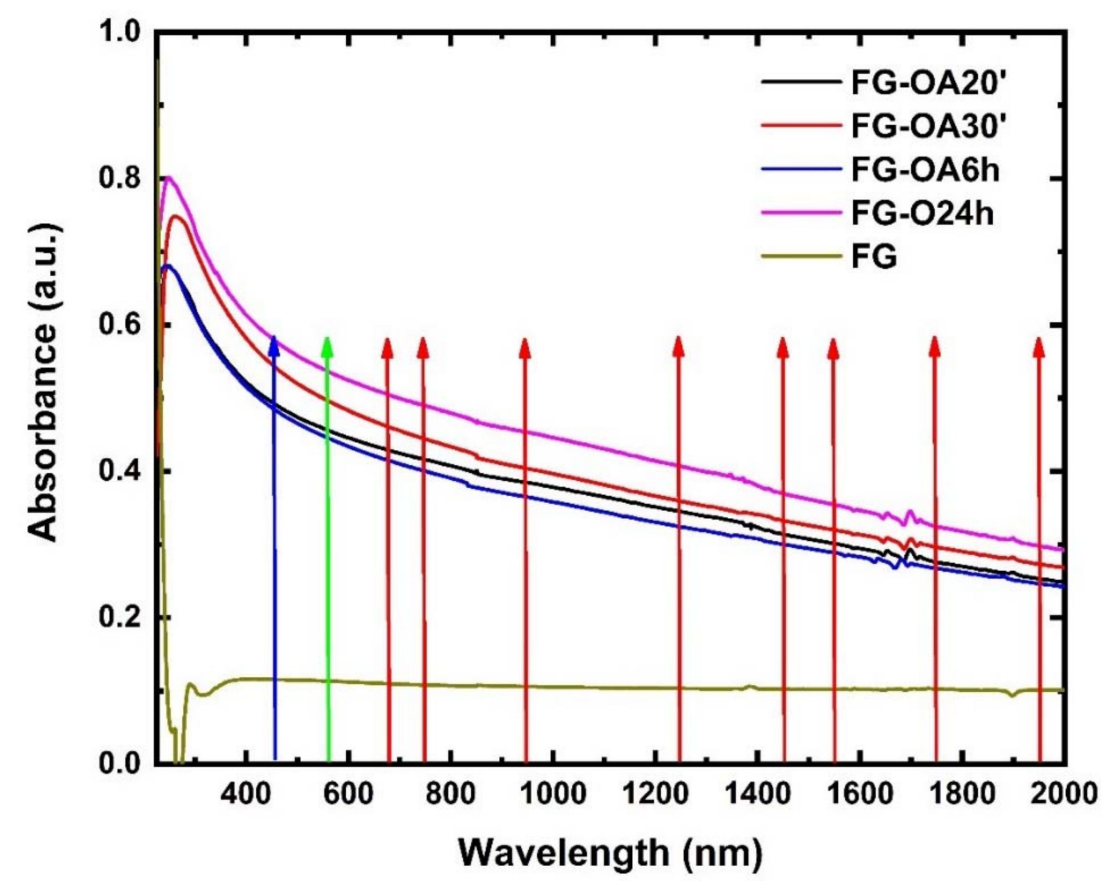

Figure 1. UV-Vis-NIR absorption spectra of pristine FG and the FG-OAx derivatives (all dispersed in DCM). 
For the determination of the nonlinear optical parameters (i.e., $\beta$ and $\gamma^{\prime}$ ) of the investigated FG-OAxs, both "open-" (OA) and "closed-aperture" (CA) Z-scan measurements were performed using FG-OAx dispersions of various concentrations, ranging from 0.01 to $0.4 \mathrm{mg} \mathrm{ml}^{-1}$. The measurements were conducted under several incident laser intensities (ranging from 0.86 to $111 \mathrm{MW} \mathrm{cm}^{-2}$ ). The corresponding "divided" Z-scans were prepared. The OA and the "divided" Z-scans were used to deduce the nonlinear absorption parameter $\beta$ and the nonlinear refractive index parameter $\gamma^{\prime}$, respectively, of the sample. In Figure 2, some representative OA and "divided" Z-scan recordings obtained under $4 \mathrm{~ns} 532 \mathrm{~nm}(\mathrm{a}, \mathrm{c})$ and $1064 \mathrm{~nm}(\mathrm{~b}, \mathrm{~d})$ laser excitation are presented. Since the solvent (i.e., DCM) exhibits a negligible NLO response under the present experimental conditions, the shown "open-“and "divided" Z-scan recordings of Figure 2 reveal directly the NLO response of the FG-OAxs. All OA Z-scan curves exhibited a transmission minimum, indicative of reverse saturable absorption (RSA) behavior, corresponding to a positive sign nonlinear absorption coefficient $\left(\beta>0\right.$ or $\left.\operatorname{Im} \chi^{(3)}>0\right)$. In fact, all the FG derivatives were found to exhibit RSA behavior, under all the experimental conditions used (i.e., incident laser intensity and dispersion concentrations), in both the visible and infrared excitation regimes. In principle, such an RSA response can be due to two-photon absorption (2PA) or excited state absorption (ESA) [24]. Further discussion about the origin of the RSA behavior is presented below the optical limiting action results.

The magnitude and the sign of the nonlinear refractive index parameter $\gamma^{\prime}$ were determined from the corresponding "divided" Z-scans. All FG-OAxs were found exhibiting a valley-peak transmission configuration, thus indicating the self-focusing behavior corresponding to $\gamma^{\prime}>0$, or equivalently to $\operatorname{Re} \chi^{(3)}>0$, under all the experimental conditions employed (Figure 2). In order to avoid the formation of cumulative thermal effects, all experiments were performed with a $1 \mathrm{~Hz}$ repetition rate of the laser.

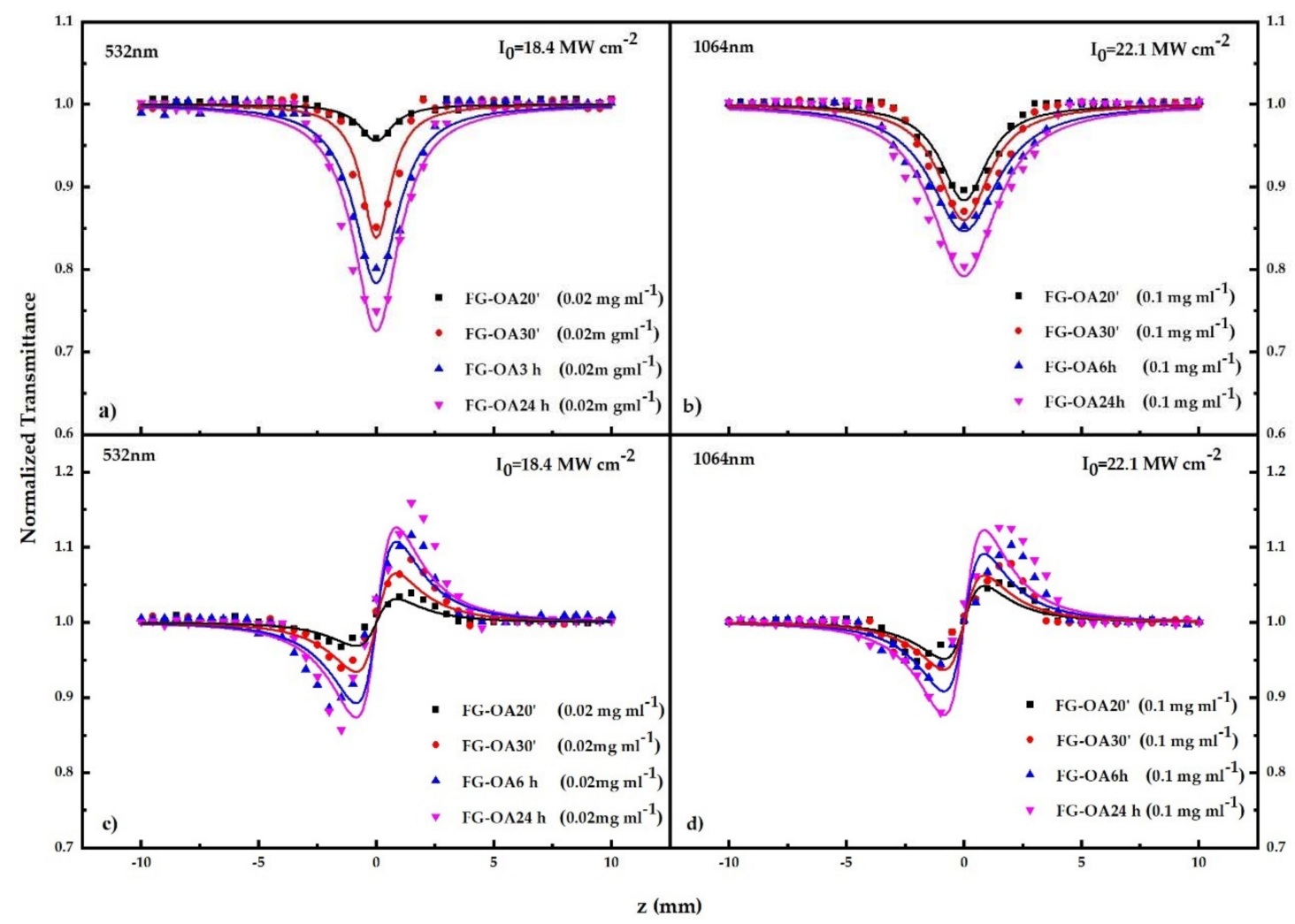

Figure 2. (a,b) “Open-aperture" (OA) and (c,d) "divided" (CA) Z-scans obtained under 4 ns 532 and $1064 \mathrm{~nm}$ laser excitations.

Following the standard Z-scan data analysis procedures [25], the NLO parameters $\beta$ and $\gamma^{\prime}$ and the third-order nonlinear susceptibility $\chi^{(3)}$ were determined, and they are summarized in Table 1. To make comparisons easier, all the shown values refer to a concentration of $1 \mathrm{mg} \mathrm{ml}^{-1}$. 
Table 1. NLO parameters of the different octylamine-functionalized FGs.

\begin{tabular}{|c|c|c|c|c|c|c|}
\hline $\begin{array}{l}\text { Wavelength } \\
(\mathrm{nm})\end{array}$ & Sample & $\begin{array}{c}\text { B } \\
\left(\times 10^{-11} \mathrm{~m} \mathrm{~W}^{-1}\right)\end{array}$ & $\begin{array}{c}\gamma^{\prime} \\
\left(\times 10^{-18} \mathrm{~m}^{2} \mathrm{~W}^{-1}\right)\end{array}$ & $\begin{array}{c}\operatorname{Im} \chi^{(3)} \\
\left(\times 10^{-13} \text { esu }\right)\end{array}$ & $\begin{array}{c}\operatorname{Re} \chi^{(3)} \\
\left(\times 10^{-13} \text { esu }\right)\end{array}$ & $\begin{array}{c}|x|^{(3)} \\
\left(\times 10^{-13} \mathrm{esu}\right)\end{array}$ \\
\hline \multirow{5}{*}{$532 \mathrm{~nm}$} & FG & $119 \pm 19$ & $-135 \pm 31$ & $62 \pm 12$ & $-173 \pm 38$ & $184 \pm 40$ \\
\hline & FG-OA 20' & $1720 \pm 175$ & $1325 \pm 235$ & $900 \pm 95$ & $1695 \pm 300$ & $1920 \pm 315$ \\
\hline & FG-OA 30' & $5010 \pm 1050$ & $1680 \pm 230$ & $2640 \pm 550$ & $2120 \pm 460$ & $3380 \pm 720$ \\
\hline & FG-OA 6 h & $7550 \pm 1300$ & $4275 \pm 425$ & $3950 \pm 650$ & $5475 \pm 550$ & $6725 \pm 770$ \\
\hline & FG-OA 24 h & $11,363 \pm 663$ & $4252 \pm 600$ & $5950 \pm 350$ & $5800 \pm 775$ & $8325 \pm 856$ \\
\hline \multirow{5}{*}{$1064 \mathrm{~nm}$} & FG & - & - & - & - & - \\
\hline & FG-OA 20' & $872 \pm 55$ & $530 \pm 100$ & $943 \pm 59$ & $676 \pm 128$ & $1160 \pm 142$ \\
\hline & FG-OA 30' & $2263 \pm 345$ & $1193 \pm 198$ & $2448 \pm 372$ & $1523 \pm 350$ & $2885 \pm 450$ \\
\hline & FG-OA 6 h & $3063 \pm 200$ & $2648 \pm 200$ & $3313 \pm 250$ & $3350 \pm 250$ & $4713 \pm 475$ \\
\hline & FG-OA $24 \mathrm{~h}$ & $6425 \pm 388$ & $3475 \pm 330$ & $6950 \pm 425$ & $4575 \pm 725$ & $8313 \pm 750$ \\
\hline
\end{tabular}

Regarding the effect of the degree of FG functionalization on the nonlinear absorption of the FG-OAxs, a dramatic enhancement is observed as the reaction of FG with $N$-octylamine proceeds, at both excitation wavelengths, as can be seen from the evolution of the $\beta$ (or $\operatorname{Im} \chi^{(3)}$ ) values in Table 1. More specifically, the nonlinear absorption coefficient $\beta$ was found to attain a maximum after $24 \mathrm{~h}$ of reaction, the FG-OA24 exhibiting an almost 100-fold increase compared to FG, for visible excitation. Interestingly, as far as concerns infrared excitation at $1064 \mathrm{~nm}$, where FG exhibits insignificant NLO absorption, this enhancement was much more pronounced, attaining a 6300-fold increase. To understand the observed enhancement of the nonlinear absorption, the different bonding configurations that nitrogen creates in the graphenic lattice have to be considered [26]. The most usual bonding configurations reported are the following: the graphitic- $\mathrm{N}$ bonding, where an $\mathrm{N}$ atom bonds to three $\mathrm{C}$ atoms directly, eliminating one $\mathrm{C}$ atom; the pyridinic-N bonding, where a six-membered ring is formed as an $\mathrm{N}$-atom is accompanied by a vacancy and bonds to two $\mathrm{C}$ atoms, and the pyrrolic-N bonding, where a nitrogen atom bonds to two carbon atoms formatting a five-membered ring. Among these bonding configurations, graphitic- $\mathrm{N}$ and pyridinic-N bondings are of $s p^{2}$ hybridization, while the pyrrolic-N one is of $s p^{3}$ hybridization. As reported by Zaoralová et al., DFT calculations showed that $\mathrm{N}$ incorporation into the carbon lattice can mostly occur at the several types of vacancies present in pristine FG, with nitrogen atoms assigned mainly in the case of pyridinic and pyrrolic configurations [12]. The presence of these configurations was further confirmed by high-resolution X-ray photoelectron spectroscopy. The corresponding results are presented in Figure 3a, where the deconvoluted N1s envelope is shown (pyrrolic $400 \mathrm{eV}$, pyridinic $398.5 \mathrm{eV}$ ). Figure $3 \mathrm{~b}$ shows the total atomic nitrogen content in the samples and atomic contents of the different nitrogen components.

In Figure $3 c$ the Raman spectra of the FG-OAxs are presented. As shown, all FG-OAxs exhibited broad D and G bands and a flat 2D region (around 2800-2900 $\mathrm{cm}^{-1}$ ). These findings suggest the presence of a large number of defects as well as functional groups, which disturb the hexagonal network of graphene. As a result, the pure graphenic $\mathrm{sp}^{2}$ domains are smaller in size, as supported by the observed suppression of the $2 \mathrm{D}$ band. In addition, as the time of reaction increases, a broadening of the D band of the FG-OAxs is observed, as can be seen in the Table 2 below. This evidence can be attributed to the increasing number of defects [27].

Table 2. Broadening of the D band of FG-OAxs.

\begin{tabular}{ccc}
\hline Sample & $\mathbf{I}_{\mathbf{D}} \mathbf{~ c m}^{\mathbf{- 1}}$ & FWHM $\mathbf{I}_{\mathbf{D}} \mathbf{~ c m}^{\mathbf{- 1}}$ \\
\hline $30 \mathrm{~min}$ & 1325 & 103 \\
$1 \mathrm{~h}$ & 1316 & 106 \\
$6 \mathrm{~h}$ & 1315 & 109 \\
$24 \mathrm{~h}$ & 1330 & 118 \\
\hline
\end{tabular}



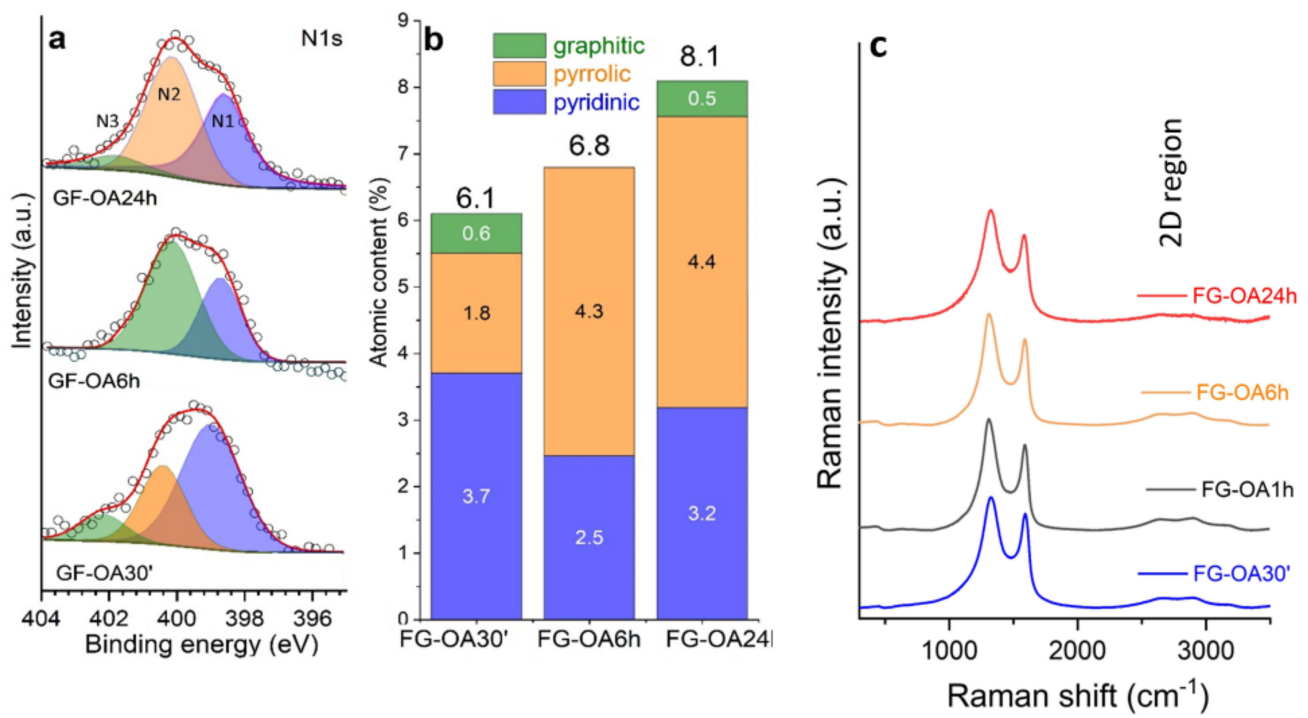

Figure 3. (a) High-resolution X-ray photoelectron spectroscopy of the N1s regions showing the deconvoluted nitrogen components in pyridinic (N1), pyrrolic (N2) and graphitic (N3) configurations.

(b) Total atomic nitrogen content in the samples and atomic contents of the different nitrogen components.

(c) Raman spectra.

Based on the above experimental evidence, the observed enhancement of the NLA can be attributed to the large number of defect-induced states, created by the interplay between $s p^{2} / s p^{3}$ sites (pyrollic and pyridinic configurations). In addition to these arguments, the differences between the local band gaps of the $s p^{3}$ and $s p^{2}$ domains in the graphitic structures create band edge fluctuations, with the $s p^{3}$ sites acting as tunnel barriers between the $\pi$ states of the $s p^{2}$ domains [28]. This situation results in the creation of strongly localized isolated $s p^{2}$ sites, which can also act as defects in the electronic band. As such, summarizing, it seems that as the degree of functionalization increases with the reaction time elapsed (i.e., higher $\mathrm{N}$ atom content corresponding to lower $\mathrm{F}$ atom content), the observed NLA increases, due to the increasing number of defects present in the FG-OAxs.

Concerning the NLO refractive response of the FG-OAxs, an alteration of the sign of the nonlinear refractive index parameter $\gamma^{\prime}$ was observed between FG and the FG-OAxs, as can be seen in Table 1 . This sign change can be understood by evoking the large difference in $\mathrm{F}$ atom content between the FG (i.e., $55 \%$ ) and the FG-OAxs (ranging from 33\% down to 2.3\%), as has been discussed in detail elsewhere [29].

Another parameter which contributes to the NLO response modification of FG upon N-octylamine functionalization is related to the changing nature of the $\mathrm{C}-\mathrm{F}$ bonds in these derivatives, due to the presence of the highly electronegative fluorine atoms. So, the nature of the C-F bond can vary within the FG lattice depending on the F content. Specifically, the C-F bond can be introduced in the form of covalent, ionic or semi-ionic ones, strongly depending on the $\mathrm{F}$ atom content. In fact, at low F atom contents, ionic $\mathrm{C}-\mathrm{F}$ bonding is favored, whereas increasing the $\mathrm{F}$ atom content, semi-ionic and covalent $\mathrm{C}-\mathrm{F}$ bondings prevail, as has been reported elsewhere [30]. Hence it is expected that in the present FG derivatives, both $\mathrm{C}-\mathrm{F}$ bonding configurations should be present, varying with the $\mathrm{F}$ atom content, i.e., the degree of functionalization.

Summarizing the above, it can be concluded that increasing the time of reaction between the $N$-octylamine ligand and pristine FG, the NLO response of the FG-OAxs increases greatly, both under visible and infrared laser excitation, in the latter case observing the largest enhancement of the NLO response by a factor of about 6300 times compared to FG. Furthermore, it is important to remember that the chemical functionalization of FG has an ON/OFF switching effect on the NLO response to excitation with infrared laser pulses. It is interesting to recall that in previous reports [11] concerning the study of some thiophenol- and diethylamino-modified FGs, under similar experimental conditions, 
the resulting NLO response of the FG derivatives was found to be much weaker than that found in the present study for the FG-OAxs ones. This result reveals the importance of the type of functionalization ligand of FG for the enhancement and the tailoring of the NLO response of FG derivatives.

\subsection{Optical Limiting Measurements}

Next, the optical limiting (OL) action of the FG-OAxs was assessed under different wavelength laser excitations, extending from visible (i.e., 450, 532, 650, $750 \mathrm{~nm}$ ) to NIR (i.e., 950, 1064, 1250, 1440, 1550 and $1750 \mathrm{~nm}$ ) wavelengths. The colored arrows shown in Figure 1 indicate the wavelengths at which the OL action was studied. More specifically, the OL action of the FG-OAxs was evaluated by measuring the incident laser beam energy on the sample, the outgoing laser beam energy, and calculating the corresponding laser fluences, $\mathrm{F}_{\text {in }}$ and $\mathrm{F}_{\text {out }}$, respectively, after taking into account the laser beam spot size. The laser beam energies were measured using two calibrated joulemeters, while the size of the irradiating area was measured by a CCD camera. From the curves shown in Figures $4 a, b, 5 a-c$ and $6 a-d$, presenting the variation of the $F_{\text {out }}$ versus $F_{\text {in }}$, the optical limiting onset, $\mathrm{OL}_{\mathrm{on}}$, was deduced. The $\mathrm{OL}_{\mathrm{on}}$ is defined as the value of the input laser fluence, $\mathrm{F}_{\mathrm{in}}$, at which the sample's linear transmittance starts to deviate from the Beer-Lambert law. The dashed lines indicate the linear transmittance of the sample (e.g., at the far left/right wings of the OA Z-scans for $532 / 1064 \mathrm{~nm}$ excitation, and the $\mathrm{F}_{\text {out }} / \mathrm{F}_{\text {in }}$ ratio at low $\mathrm{F}_{\text {in }}$ fluence). The obtained OL action results, for each excitation wavelength and for all FG-OAxs, are presented in Figures 4 and 5 . As can be seen in these figures, all $\mathrm{N}$-octylamine functionalized FGs exhibited a very important OL action, which was found to be increasing with the degree of functionalization (i.e., the reaction time between FG and N-octylamine). In Figure 4a,b, the OL actions of the FG-OAxs under the 532 and 1064 nm laser excitations are presented separately from the OL action studied at the other excitation wavelengths used, as the results concerning the 532 and $1064 \mathrm{~nm}$ excitation wavelengths are the most commonly reported in the related literature. The corresponding $\mathrm{OL}_{\mathrm{on}}$ values for each FG-OAx, determined from the curves of Figures 4 and 6, are presented in Table 3. It is important to remember that lower $\mathrm{OL}_{\mathrm{on}}$ values indicate more efficient OL action. From the monotonically decreasing $\mathrm{OL}_{\mathrm{on}}$ values of Table 3 , it becomes evident that as the reaction time of FG with $N$-octylamine increases, the optical limiting efficiency is greatly improved. This is attributed to the increasing number of defects, as discussed previously, leading to more important NLA, which is the principal mechanism responsible for the observed OL action exhibited by the FG-OAxs, as will be discussed in more detail in the following.
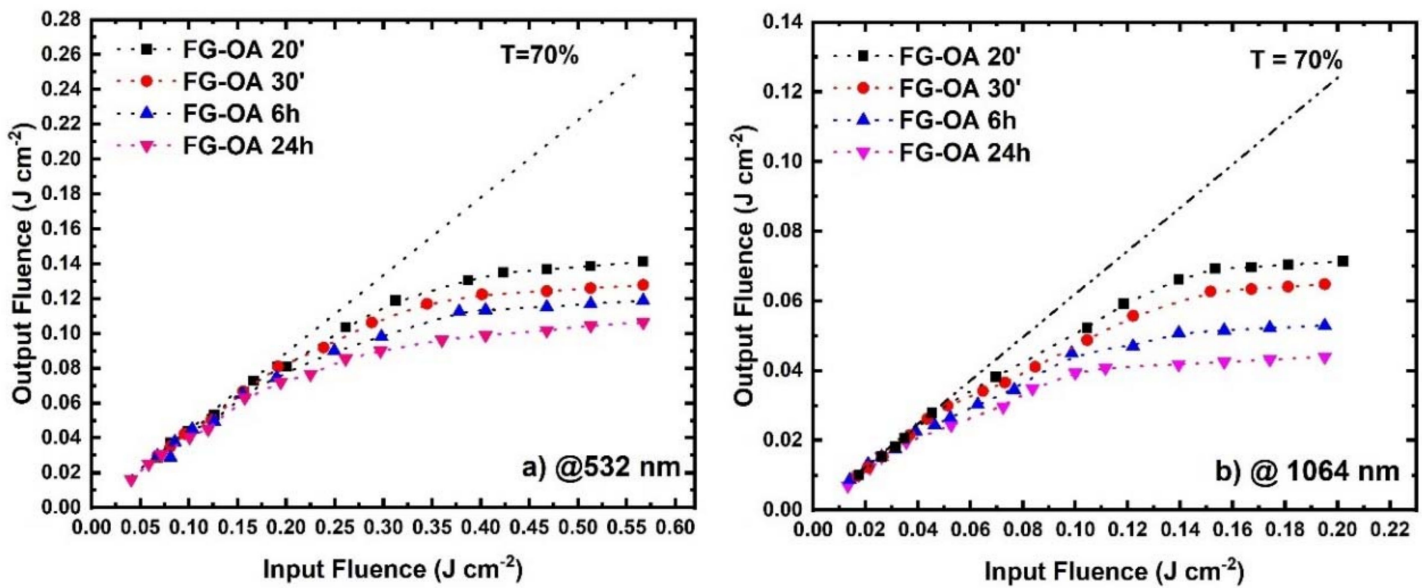

Figure 4. Optical limiting action of FG-OAxs under (a) 532 and (b)1064 nm laser irradiation.

As can be seen form this table, the $\mathrm{OL}_{\mathrm{on}}$ values of the FG-OAxs (all dispersions prepared exhibiting $70 \%$ linear transmittance at each excitation wavelength) were all found to be quite low, both under visible and infrared excitation, and in the latter case being substantially smaller. This finding suggests clearly that the FG-OAxs are much more efficient optical limiters when compared, for example, to other 
materials generally considered as benchmark materials for optical limiting, e.g., some $\mathrm{C}_{60}$-toluene solutions (with $\mathrm{OL}_{\mathrm{on}}=0.2 \mathrm{~J} \mathrm{~cm}^{-2}$ ) [31] and carbon black suspensions (with $\mathrm{OL}_{\mathrm{on}}=2.2 \mathrm{~J} \mathrm{~cm}^{-2}$ ) [32].

Then, the optical limiting action of the FG-OAx derivatives was investigated under irradiation by laser radiations of wavelengths ranging from the visible to the NIR spectral region, i.e., 450, 650, 750, 950, 1250, 1550 and $1750 \mathrm{~nm}$. In Figures 5 and 6, the obtained OL action of the FG-OAxs under the different irradiation wavelengths is presented. In all cases, efficient OL action was observed, becoming more efficient from $450 \mathrm{~nm}$ to about $1250 \mathrm{~nm}$, while becoming less efficient towards the NIR wavelengths. The corresponding $\mathrm{OL}_{\text {on }}$ values determined from the OL curves of Figures 5 and 6 are listed in Table 4.
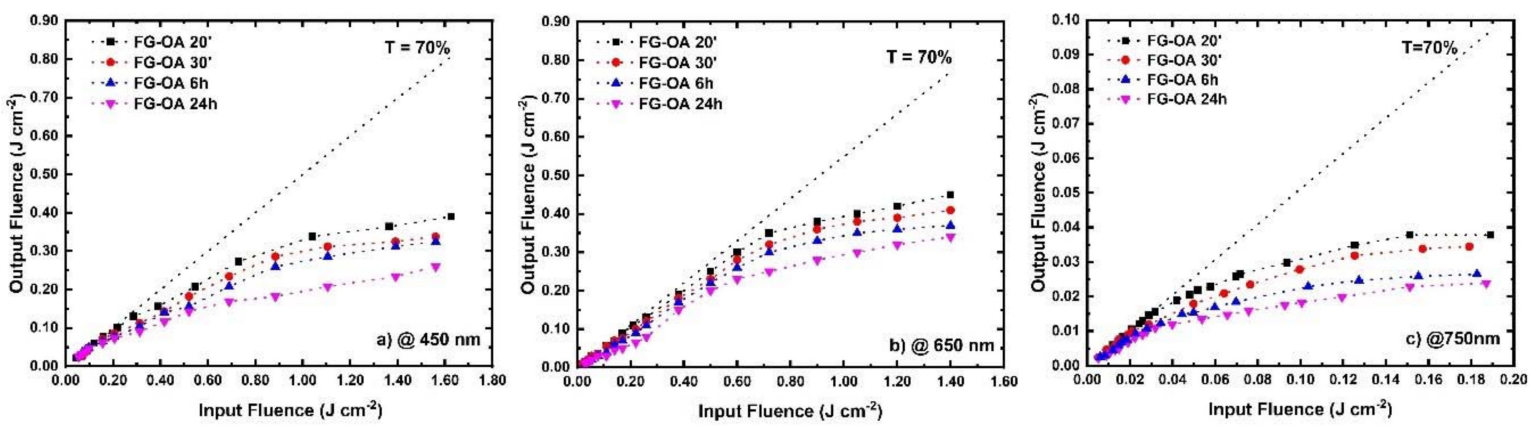

Figure 5. Optical limiting action of FG-OAxs under (a) 450, (b) 650 and (c) $750 \mathrm{~nm}$ laser irradiation.
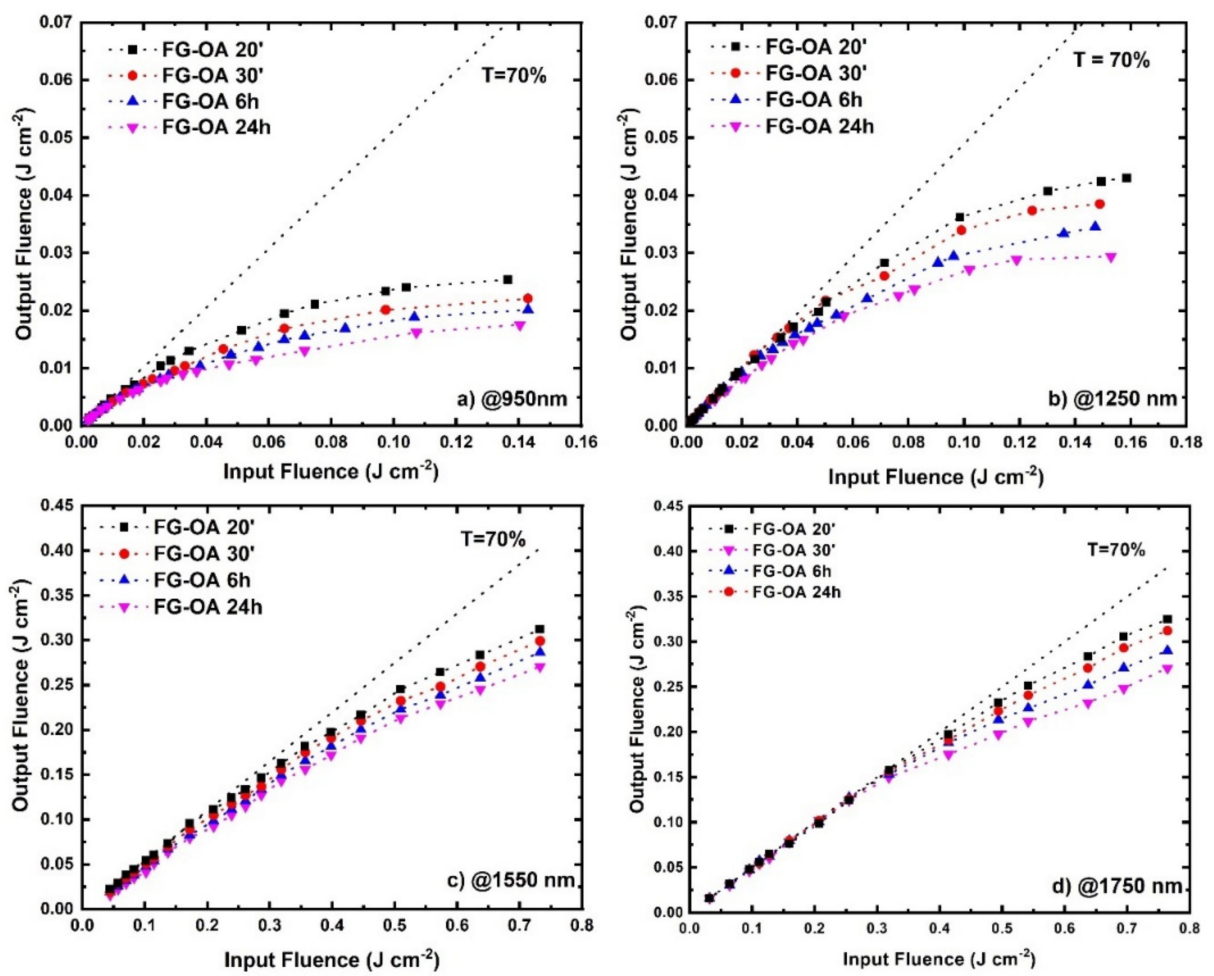

Figure 6. Optical limiting action of FG-OAxs under (a) 950, (b) 1250, (c) 1550 and (d) $1750 \mathrm{~nm}$ laser irradiation.

The variation of the $\mathrm{OL}_{\text {on }}$ values from visible to NIR wavelengths is schematically depicted in Figure 7. As shown, the $\mathrm{OL}_{\text {on }}$ values of all FG-OAxs were found to be decreasing towards $800 \mathrm{~nm}$, attaining a plateau in the spectral region from about 750 to $1250 \mathrm{~nm}$, then again increasing towards $1750 \mathrm{~nm}$. Specifically, the $\mathrm{OL}_{\mathrm{on}}$ values of the studied FG-OAxs were found decreasing by almost an order of magnitude, attaining very low values of about $0.01 \mathrm{~J} \mathrm{~cm}^{-2}$ at 950 and $1250 \mathrm{~nm}$. 
Table 3. Optical limiting onsets ( $\left.\mathrm{OL}_{\mathrm{on}}\right)$ of FG-OAxs under 532nm and $1064 \mathrm{~nm}$ laser irradiation.

\begin{tabular}{ccc}
\hline $\begin{array}{c}\text { Wavelength } \\
(\mathbf{n m})\end{array}$ & Sample & OL $_{\text {on }}\left(\mathbf{J ~ c m}^{-2}\right)$ \\
\hline & FG-OA 20' & 0.18 \\
532 & FG-OA 30' & 0.16 \\
& FG-OA 6 h & 0.13 \\
& FG-OA 24 h & 0.10 \\
\hline \multirow{3}{*}{1064} & FG-OA 20' & 0.06 \\
& FG-OA 30' & 0.04 \\
& FG-OA 6 h & 0.03 \\
& FG-OA 24 h & 0.02 \\
\hline
\end{tabular}

Table 4. Optical Limiting onsets of FG-OAxs under 450, 650, 750, 950, 1250, 1550 and $1750 \mathrm{~nm}$ laser irradiation.

\begin{tabular}{|c|c|c|}
\hline $\begin{array}{l}\text { Wavelength } \\
\text { (nm) }\end{array}$ & Sample & $\mathrm{OL}_{\text {on }}\left(\mathrm{J} \mathrm{cm}^{-2}\right)$ \\
\hline \multirow{4}{*}{450} & FG-OA $20^{\prime}$ & 0.21 \\
\hline & FG-OA $30^{\prime}$ & 0.20 \\
\hline & FG-OA 6 h & 0.15 \\
\hline & FG-OA $24 \mathrm{~h}$ & 0.12 \\
\hline \multirow{4}{*}{650} & FG-OA $20^{\prime}$ & 0.17 \\
\hline & FG-OA 30' & 0.14 \\
\hline & FG-OA 6 h & 0.12 \\
\hline & FG-OA 24 h & 0.09 \\
\hline \multirow{4}{*}{750} & FG-OA $20^{\prime}$ & 0.04 \\
\hline & FG-OA 30' & 0.03 \\
\hline & FG-OA $6 \mathrm{~h}$ & 0.03 \\
\hline & FG-OA $24 \mathrm{~h}$ & 0.02 \\
\hline \multirow{4}{*}{950} & FG-OA $20^{\prime}$ & 0.02 \\
\hline & FG-OA 30' & 0.02 \\
\hline & FG-OA $6 \mathrm{~h}$ & 0.02 \\
\hline & FG-OA $24 \mathrm{~h}$ & 0.01 \\
\hline \multirow{4}{*}{1250} & FG-OA $20^{\prime}$ & 0.03 \\
\hline & FG-OA 30' & 0.03 \\
\hline & FG-OA 6 h & 0.02 \\
\hline & FG-OA $24 \mathrm{~h}$ & 0.01 \\
\hline \multirow{4}{*}{1550} & FG-OA $20^{\prime}$ & 0.20 \\
\hline & FG-OA 30' & 0.16 \\
\hline & FG-OA 6 h & 0.10 \\
\hline & FG-OA $24 \mathrm{~h}$ & 0.07 \\
\hline \multirow{4}{*}{1750} & FG-OA 20' & 0.33 \\
\hline & FG-OA $30^{\prime}$ & 0.30 \\
\hline & FG-OA 6 h & 0.28 \\
\hline & FG-OA $24 \mathrm{~h}$ & 0.26 \\
\hline
\end{tabular}

Compared to other 2D nanomaterials, the studied FG-OAxs are found to exhibit a much stronger OL behavior. So, in a recent work [33] studying the OL of graphene and some transition metal dichalcogenides-TMDCs (e.g., $\mathrm{MoS}_{2}, \mathrm{MoSe}_{2}, \mathrm{WS}_{2}$, and $\mathrm{WSe}_{2}$ ) nanosheet dispersions under 6 ns 532 and $1064 \mathrm{~nm}$ laser excitations, it was reported that graphene exhibited the lowest onset of OL, i.e., $\sim 0.44$ and $\sim 0.64 \mathrm{~J} \mathrm{~cm}^{-2}$, respectively, whereas the TMDC dispersions exhibited $\mathrm{OL}_{\text {on }}$ values larger than 1 and $1.37 \mathrm{~J} \mathrm{~cm}^{-2}$, respectively. In another work [34], concerning the OL action of some graphene nanosheets (GNS), graphene nanoribbons (GNR), graphene oxide nanosheets (GONS) and graphene 
oxide nanoribbons (GONR), it was found that all these derivatives exhibited $\mathrm{OL}_{\text {on }}$ values ranging between 0.5 and $3 \mathrm{~J} \mathrm{~cm}^{-2}$ for visible excitation, whereas for infrared excitation the $\mathrm{OL}_{\mathrm{on}}$ was found to be significantly larger, i.e., larger than $3.5 \mathrm{~J} \mathrm{~cm}^{-2}$. So, in all cases, the FG-OAxs studied here exhibited much more effective optical limiting.

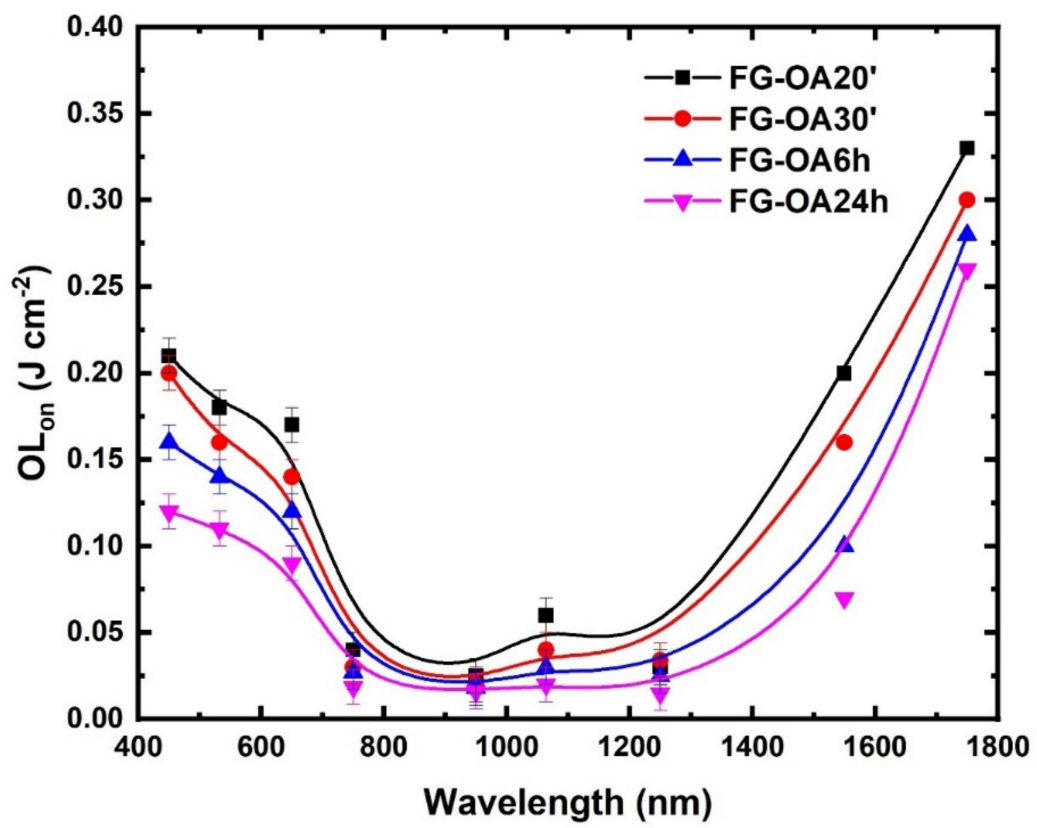

Figure 7. Variation of the optical limiting onset $\left(\mathrm{OL}_{\mathrm{on}}\right)$ of the FG-OAx derivatives with the irradiation wavelength.

Regarding the OL action of some other fluorographene derivatives studied by our group, such as some thiophenol-modified fluorographenes [11], the present $N$-octylamine-modified fluorographenes were found to exhibit comparable OL action. More specifically, the thiophenol fluorographenes exhibited $\mathrm{OL}_{\text {on }}$ values of $\sim 0.1 \mathrm{~J} \mathrm{~cm}^{-2}$ and $\sim 0.05 \mathrm{~J} \mathrm{~cm}^{-2}$ under 532 and $1064 \mathrm{~nm}$ excitations, respectively, which are similar to the $\mathrm{OL}_{\text {on }}$ values of FG-OA $24 \mathrm{~h}$ and FG-OA $6 \mathrm{~h}$ derivatives. In another work [13] studying the OL action of a diethylamino-modified fluorographene (CDEA), it was found that its $\mathrm{OL}_{\text {on }}$ was increasing towards the NIR wavelengths. In particular, the $\mathrm{OL}_{\text {on }}$ of CDEA was found to be $\sim 0.045 \mathrm{~J} \mathrm{~cm}^{-2}$ at $532 \mathrm{~nm}$, increasing continuously and reaching the value of $\sim 0.16 \mathrm{~J} \mathrm{~cm}^{-2}$ at $1750 \mathrm{~nm}$. So, in this case, the present FG-OAxs exhibit slightly weaker OL values at both 532 and $1750 \mathrm{~nm}$. However, the FG-OAxs were found to exhibit significantly stronger OL values in the region 750-1250 nm, with their $\mathrm{OL}_{\text {on }}$ being over an order of magnitude lower than that of CDEA.

In general, there are different processes that can give rise to an optical limiting action. Some of the processes occurring under ns laser irradiation include the nonlinear absorption (NLA), the nonlinear scattering (NLS) and the induced thermal scattering (ITS). As far as concerns the last two processes, separate measurements were performed to evaluate their contributions to the observed OL action. So, at first, the presence of NLS was examined. In that view, experiments were conducted whereby the sample was irradiated by the laser beam while a sensitive photodiode was placed behind it, positioned at different directions with respect to the laser beam propagation direction. In front of the photodiode, a slit was placed to minimize the light due to reflections of the cell walls, ambient light, etc. However, for the range of laser energies used, negligible NLS was detected for all FG-OAx samples.

Next, the presence of scattered light originating from ITS-related phenomena has been explored. In this case, the formation of scattering centers (e.g., bubbles) in the sample due to heating of the surrounding medium can occur. As it has been reported elsewhere (e.g., [35,36]), ns laser pulses can result in the efficient heating of the graphene flakes. This excess heating can be efficiently dissipated to the surrounding solvent, giving rise to the formation of solvent bubbles, which expand quickly at the 
vapor-solution interface, acting as efficient scattering centers. In addition to this, powerful enough laser beams can induce micro-plasmas, which can also operate as scattering centers. To investigate such ITS phenomena in the present measurements, the unfocused beam of a Helium-Neon (He-Ne) laser was used, crossing the focal volume of the focused Nd:YAG laser beam. The projection of the He-Ne beam onto a white screen placed a few meters away from the sample allows the visual observation of bubble formation. Although no such phenomena were observed for the laser energies employed in the present study, for precaution reasons, all experiments were carried out with operating the lasers at a $1 \mathrm{~Hz}$ repetition rate.

Finally, the mechanism responsible for the nonlinear absorption of the studied FG-OAxs was investigated. As mentioned in the previous section, for the Z-scan measurements carried out under 532 and $1064 \mathrm{~nm}$ excitations, they were all found exhibiting valley-shaped "open-aperture" Z-scans, corresponding to RSA behavior, implying the presence of nonlinear absorption. However, depending on the excitation wavelength, the pulse duration and the material properties, the observed nonlinear absorption can arise from two-photon absorption (2PA) and/or excited state absorption (ESA). To evaluate the nonlinear absorption coefficient $\beta$, the OA Z-scans were fitted by the following equation, described in detail elsewhere [37]:

$$
T(x)=\frac{1}{\sqrt{\pi}\left(\frac{\beta I_{0} L_{e f f}}{1+[x]^{2}}\right)} \int_{-\infty}^{+\infty} \ln \left[1+\frac{\beta I_{0} L_{e f f}}{1+[x]^{2}} e^{-t}\right] d t
$$

This equation implies nonlinear absorption due to two-photon absorption (2PA). As can be seen from the solid lines of the OA Z-scan curves, corresponding to the best fit by this equation, a very successful fitting was obtained, suggesting that the 2PA process is the main mechanism giving rise to the RSA behavior.

Furthermore, the determined nonlinear absorption coefficient, $\beta$, was plotted as a function of the on-axis peak intensity for all FG-OAxs. If the ESA process is operating, it should cause the depletion of the ground state, leading to variations of the $\beta$ with the on-axis peak intensity [38]. However, as illustrated in Figure $8 \mathrm{a}, \mathrm{b}$, the $\beta$ was found to be independent of the on-axis peak intensity, under both the 532 and the $1064 \mathrm{~nm}$ excitation, thus suggesting that any contribution of the ESA to the nonlinear absorption should be negligible. Therefore, it can be safely concluded that 2PA seems to be the principal mechanism of the observed OL action.
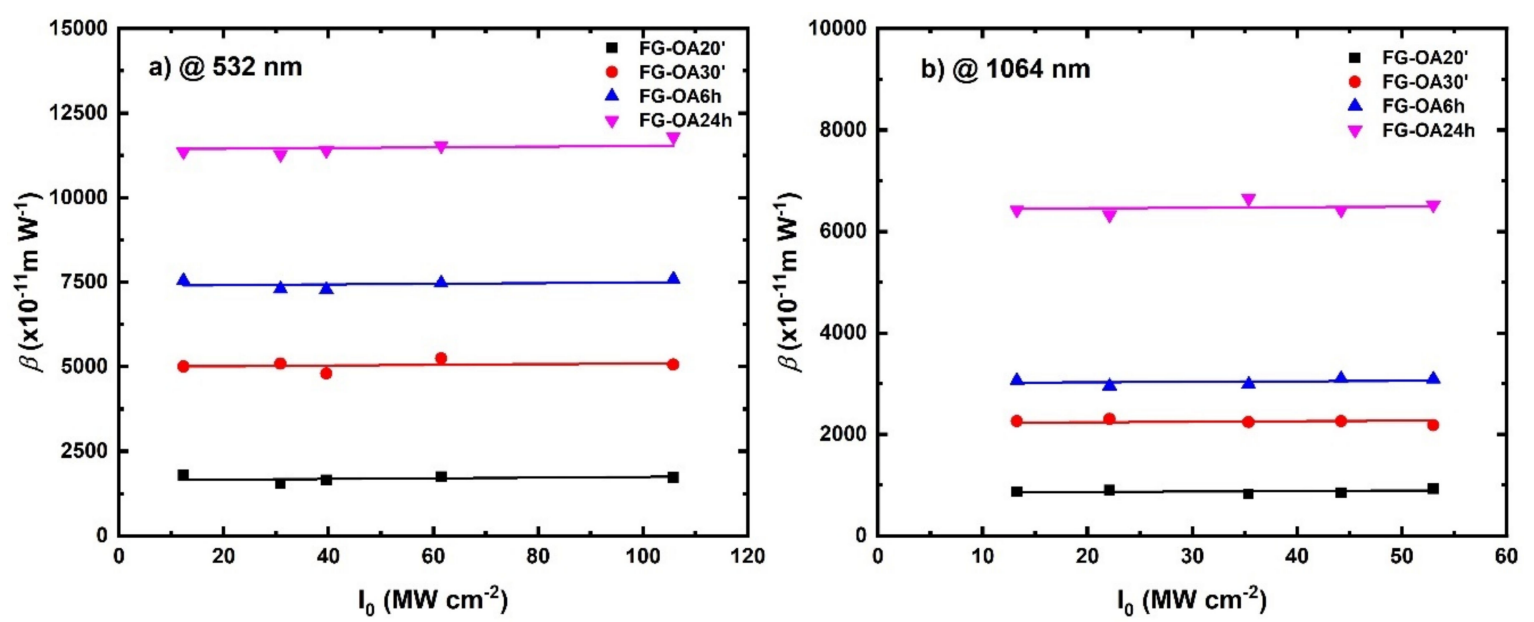

Figure 8. Dependence of the nonlinear absorption coefficient $\beta$ upon the on-axis peak intensity for (a) $532 \mathrm{~nm}$ and (b) $1064 \mathrm{~nm}$ laser excitation. 


\section{Conclusions}

In conclusion, pristine fluorographene (FG) and four FG derivatives (FG-OAx), prepared after the partial nucleophilic substitution of FG with $N$-octylamine and having different degrees of functionalization, were studied. The NLO responses of the FG and the FG-OAx derivatives were investigated by means of the Z-scan technique, along with their OL action, under various excitation wavelengths ranging from visible $(450 \mathrm{~nm})$ to NIR $(1750 \mathrm{~nm})$. Their NLO response was turned ON shortly after the initiation of the partial nucleophilic substitution of the FG, leading to strong enhancement with the time of reaction. The FG-OAxs were found to be exhibiting strong nonlinear absorptions, attributed to defect-induced states due to the $\mathrm{C}-\mathrm{N}$ bonding configuration formed by the incorporation of nitrogen into the graphenic lattice. Their strong nonlinear absorption was found to result in extremely efficient OL action, rendering the FG-OAxs very efficient optical limiters suitable to and attractive for a plethora of OL applications, from the visible to NIR spectral regions. The above experimental findings demonstrate clearly that FG is a useful platform for achieving chemical functionalization, leading to a wide portfolio of fluorographene derivatives, while by controlling the degree of functionalization the nonlinear optical properties of the derivatives can be largely tuned, providing materials with tailored properties throughout almost the entire optical spectrum, which are highly desirable for several photonic and optoelectronic applications.

Author Contributions: Conceptualization, S.C.; methodology, A.S., M.S., I.P. and I.O.; software, A.S., M.S., I.P. and I.O.; validation, A.S., M.S., I.P. and I.O.; formal analysis, A.S., M.S., I.P. and I.O.; investigation, A.S., M.S., I.P. and I.O.; resources, S.C. and I.O.; data curation, A.S., M.S., I.P. and I.O.; writing-original draft preparation, A.S.; writing-review and editing, A.S., I.P., I.O. and S.C.; visualization, A.S., I.P. and I.O.; supervision, S.C.; project administration, S.C.; funding acquisition, S.C. All authors have read and agreed to the published version of the manuscript.

Funding: This research received no external funding.

Acknowledgments: A.S., M.S., I.P. and S.C. acknowledge support by the project «HELLAS-CH» in the frame of «Cooperation ELI-LASERLAB Europe, HiPER \& IPERION-CH.gr» (MIS 5002735) which is implemented under the "Action for the Strategic Development on the Research and Technological Sector", funded by the Operational Programme "Competitiveness, Entrepreneurship and Innovation" (NSRF 2014-2020) and co-financed by Greece and the European Union (European Regional Development Fund). I.P. acknowledges support from the Hellenic Foundation for Research and Innovation (HFRI) under the HFRI PhD Fellowship grant (Number: 80997). I.O. acknowledges the support by ERDF/ESF “Nano4Future" (No. CZ.02.1.01/0.0/0.0/16_019/0000754). Aristides Bakandritsos is warmly acknowledged for useful discussions and suggestions during the course of this work. We also thank Kateřina Štymplová for collecting the Raman spectra.

Conflicts of Interest: The authors declare no conflict of interest.

\section{References}

1. Nair, R.R.; Ren, W.C.; Jalil, R.; Riaz, I.; Kravets, V.G.; Britnell, L.; Blake, P.; Schedin, F.; Mayorov, A.S.; Yuan, S.J.; et al. Fluorographene: A Two-Dimensional Counterpart of Teflon. Small 2010, 6, 2877-2884. [CrossRef] [PubMed]

2. Zbořil, R.; Karlický, F.; Bourlinos, A.B.; Steriotis, T.A.; Stubos, A.K.; Georgakilas, V.; Šafářová, K.; Jančík, D.; Trapalis, C.; Otyepka, M. Graphene Fluoride: A Stable Stoichiometric Graphene Derivative and Its Chemical Conversion to Graphene. Small 2010, 6, 2885-2891. [CrossRef] [PubMed]

3. Whitener, K.E.; Stine, R.; Robinson, J.T.; Sheehan, P.E. Graphene as Electrophile: Reactions of Graphene Fluoride. J. Phys. Chem. C 2015, 119, 10507-10512. [CrossRef]

4. Bakandritsos, A.; Pykal, M.; Blonski, P.; Jakubec, P.; Chronopoulos, D.D.; Polakova, K.; Georgakilas, V.; Cepe, K.; Tomanec, O.; Ranc, V.; et al. Cyanographene and Graphene Acid: Emerging Derivatives Enabling High-Yield and Selective Functionalization of Graphene. ACS Nano 2017, 11, 2982-2991. [CrossRef]

5. Urbanova, V.; Hola, K.; Bourlinos, A.B.; Cepe, K.; Ambrosi, A.; Loo, A.H.; Pumera, M.; Karlicky, F.; Otyepka, M.; Zboril, R. Thiofluorographene-Hydrophilic Graphene Derivative with Semiconducting and Genosensing Properties. Adv. Mater. 2015, 27, 2305-2310. [CrossRef] 
6. Urbanova, V.; Karlicky, F.; Matej, A.; Sembera, F.; Janousek, Z.; Perman, J.A.; Ranc, V.; Cepe, K.; Michl, J.; Otyepka, M.; et al. Fluorinated Gaphenes as Advanced Biosensors-Effect of Fluorine Coverage on Electron Transfer Properties and Adsorption of Biomolecules. Nanoscale 2016, 8, 12134-12142. [CrossRef]

7. Liang, S.-Z.; Chen, G.; Harutyunyan, A.R.; Cole, M.W.; Sofo, J.O. Analysis and Optimization of Carbon Nanotubes and Graphene Sensors Based on Adsorption-Desorption Kinetics. Appl. Phys. Lett. 2013, 103, 233108. [CrossRef]

8. Das, S.; Sudhagar, P.; Verma, V.; Song, D.; Ito, E.; Lee, S.Y.; Kang, Y.S.; Choi, W. Amplifying Charge-Transfer Characteristics of Graphene for Triiodide Reduction in Dye-Sensitized Solar Cells. Adv. Funct. Mater. 2011, 21, 3729-3736. [CrossRef]

9. Worsley, K.A.; Ramesh, P.; Mandal, S.K.; Niyogi, S.; Itkis, M.E.; Haddon, R.C. Soluble Graphene Derived from Graphite Fluoride. Chem. Phys. Lett. 2007, 445, 51-56. [CrossRef]

10. Dubecky, M.; Otyepkova, E.; Lazar, P.; Karlicky, F.; Petr, M.; Cepe, K.; Banas, P.; Zboril, R.; Otyepka, M. Reactivity of Fluorographene: A Facile Way toward Graphene Derivatives. J. Phys. Chem. Lett. 2015, 6, 1430-1434. [CrossRef]

11. Stathis, A.; Papadakis, I.; Karampitsos, N.; Couris, S.; Potsi, G.; Bourlinos, A.B.; Otyepka, M.; Zboril, R. Thiophenol-Modified Fluorographene Derivatives for Nonlinear Optical Applications. ChemPlusChem 2019, 84, 1288-1298. [CrossRef] [PubMed]

12. Zaoralová, D.; Hrubý, V.; Šedajová, V.; Mach, R.; Kupka, V.; Ugolotti, J.; Bakandritsos, A.; Medved', M.; Otyepka, M. Tunable Synthesis of Nitrogen Doped Graphene from Fluorographene under Mild Conditions. ACS Sustain. Chem. Eng. 2020, 8, 4764-4772. [CrossRef]

13. Papadakis, I.; Stathis, A.; Bourlinos, A.B.; Couris, S. Diethylamino-Fluorographene: A 2D Material with Broadband and Efficient Optical Limiting Performance (from 500 to $1800 \mathrm{~nm}$ ) with Very Large Nonlinear Optical Response. Nano Select. 2020, 1, 395-404. [CrossRef]

14. Li, X.L.; Wang, X.R.; Zhang, L.; Lee, S.W.; Dai, H.J. Chemically Derived, Ultrasmooth Graphene Nanoribbon Semiconductors. Science 2008, 319, 1229-1232. [CrossRef] [PubMed]

15. Wang, X.R.; Li, X.L.; Zhang, L.; Yoon, Y.; Weber, P.K.; Wang, H.L.; Guo, J.; Dai, H.J. N-Doping of Graphene through Electrothermal Reactions with Ammonia. Science 2009, 324, 768-771. [CrossRef]

16. Tuček, J.; Holá, K.; Bourlinos, A.B.; Błoński, P.; Bakandritsos, A.; Ugolotti, J.; Dubecký, M.; Karlický, F.; Ranc, V.; Čépe, K.; et al. Room Temperature Organic Magnets Derived from sp ${ }^{3}$ Functionalized Graphene. Nat. Commun. 2017, 8, 14525. [CrossRef]

17. Matochová, D.; Medved', M.; Bakandritsos, A.; Steklý, T.; Zbořil, R.; Otyepka, M. 2D Chemistry: Chemical Control of Graphene Derivatization. J. Phys. Chem. Lett. 2018, 9, 3580-3585. [CrossRef]

18. Stathis, A.; Stavrou, M.; Papadakis, I.; Bakandritsos, A.; Steklý, T.; Otyepka, M.; Couris, S. Octylamine Modified Fluorographenes as a Versatile Platform for the Efficient Engineering of the Nonlinear Optical Properties of Fluorinated Graphenes. Adv. Photonics Res. 2020. accepted. [CrossRef]

19. Medved', M.; Zoppellaro, G.; Ugolotti, J.; Matochová, D.; Lazar, P.; Pospíšil, T.; Bakandritsos, A.; Tuček, J.; Zbořil, R.; Otyepka, M. Reactivity of Fluorographene is Triggered by Point Defects: Beyond the Perfect 2D World. Nanoscale 2018, 10, 4696-4707. [CrossRef]

20. Sheik-Bahae, M.; Said, A.A.; Wei, T.H.; Hagan, D.J.; Van Stryland, E.W. Sensitive Measurement of Optical Nonlinearities Using a Single Beam. IEEE J. Quant. Electr. 1990, 26, 760-769. [CrossRef]

21. Papagianouli, I.; Iliopoulos, K.; Gindre, D.; Sahraoui, B.; Krupka, O.; Smokal, V.; Kolendo, A.; Couris, S. Third-Order Nonlinear Optical Response of Push-Pull Zzobenzene Polymers. Chem. Phys. Lett. 2012, 554, 107-112. [CrossRef]

22. Li, D.; Müller, M.B.; Gilje, S.; Kaner, R.B.; Wallace, G.G. Processable Aqueous Dispersions of Graphene Nanosheets. Nat. Nanotechnol. 2008, 3, 101-105. [CrossRef] [PubMed]

23. Potsi, G.; Bourlinos, A.B.; Mouselimis, V.; Polakova, K.; Chalmpes, N.; Gournis, D.; Kalytchuk, S.; Tomanec, O.; Błonski, P.; Medved', M.; et al. Intrinsic Photo-Luminescence of Amine-Functionalized Graphene Derivatives for Bioimaging Applications. Appl. Mater. Today 2019, 17, 112-122. [CrossRef]

24. Sutherland, R.L. Handbook of Nonlinear Optics, 2nd ed.; Dekker: New York, NY, USA, 2003; pp. $612-615$.

25. Couris, S.; Koudoumas, E.; Ruth, A.A.; Leach, S. Concentration and Wavelength Dependence of the Effective Third-Order Susceptibility and Optical Limiting of $\mathrm{C}_{60}$ in Toluene Solutions. J. Phys. B At. Mol. Opt. Phys. 1995, 28, 4537. [CrossRef] 
26. Yadav, R.; Dixit, C.K. Synthesis, Characterization and Potential application of Nitrogen-doped Graphene. J. Sci. Adv. Mater. Devices 2017, 2, 141-149. [CrossRef]

27. Ferrari, A.C.; Robertson, J. Interpretation of Raman Spectra of Disordered and Amorphous Carbon. Phys. Rev. B 2000, 61, 14095-14107. [CrossRef]

28. Jiang, X.-F.; Polavarapu, L.; Neo, S.T.; Venkatesan, T.; Xu, Q.-H.J. Tunable Broadband Nonlinear Optical Properties of Black Phosphorus Quantum Dots for Femtosecond Laser Pulses. Phys. Chem. Lett. 2012, 3, 785-790. [CrossRef]

29. Liaros, N.; Bourlinos, A.B.; Zboril, R.; Couris, S. Fluoro-Graphene: Nonlinear Optical Properties. Opt. Express 2013, 21, 21027. [CrossRef]

30. Papadakis, I.; Bouza, Z.; Couris, S.; Bourlinos, A.B.; Mouselimis, V.; Kouloumpis, A.; Gournis, D.; Bakandritsos, A.; Ugolotti, J.; Zboril, R. Hydrogenated Gluorographene: A 2D Counterpart of Graphene with Enhanced Nonlinear Optical Properties. J. Phys. Chem. C 2017, 121, 22567-22575. [CrossRef]

31. Liaros, N.; Aloukos, P.; Kolokithas-Ntoukas, A.; Bakandritsos, A.; Szabo, T.; Zboril, R.; Couris, S. Nonlinear Optical Properties and Broadband Optical Power Limiting Action of Graphene Oxide Colloids. J. Phys. Chem. C 2013, 117, 6842-6850. [CrossRef]

32. Chen, P.; Wu, X.; Sun, X.; Lin, J.; Ji, W.; Tan, K.L. Electronic Structure and Optical Limiting Behavior of Carbon Nanotubes. Phys. Rev. Lett. 1999, 82, 2548-2551. [CrossRef]

33. Dong, N.; Li, Y.; Feng, Y.; Zhang, S.; Zhang, X.; Chang, C.; Fan, J.; Zhang, L.; Wang, J. Optical Limiting and Theoretical Modelling of Layered Transition Metal Dichalcogenide Nanosheets. Sci. Rep. 2015, 5, 14646. [CrossRef] [PubMed]

34. Feng, M.; Zhan, H.; Chen, Y. Nonlinear Optical and Optical Limiting Properties of Graphene Families. Appl. Phys. Lett. 2010, 96, 033107. [CrossRef]

35. Wang, J.; Hernandez, Y.; Lotya, M.; Coleman, J.N.; Blau, W.J. Broadband Nonlinear Optical Response of Graphene Dispersions. Adv. Mater. 2009, 21, 2430-2435. [CrossRef]

36. Mansour, K.; Soileau, M.J.; Van Stryland, E.W. Nonlinear Optical Properties of Carbon-Black Suspensions (Ink). J. Opt. Soc. Am. B 1992, 9, 1100-1109. [CrossRef]

37. Liaros, N.; Orfanos, I.; Papadakis, I.; Couris, S. Nonlinear Optical Response of some Graphene Oxide and Graphene Fluoride Derivatives. Optofluid. Microfluid. Nanofluid. 2016, 3, 53-58. [CrossRef]

38. Anand, B.; Podila, R.; Ayala, P.; Oliviera, L.; Philip, R.; Siva Sankara Sai, S.; Zakhidov, A.A.; Rao, A.M. Nonlinear Optical Properties of Boron Doped Single-Walled Carbon Nanotubes. Nanoscale 2013, 5, 7271-7276. [CrossRef]

Publisher's Note: MDPI stays neutral with regard to jurisdictional claims in published maps and institutional affiliations.

(C) 2020 by the authors. Licensee MDPI, Basel, Switzerland. This article is an open access article distributed under the terms and conditions of the Creative Commons Attribution (CC BY) license (http://creativecommons.org/licenses/by/4.0/). 